\title{
DIFFERENCE INDICES OF QUASI-REGULAR DIFFERENCE ALGEBRAIC SYSTEMS
}

\section{JIE WANG}

School of Mathematical Sciences

Peking University

P. R. China

e-mail: wangjie212@mails.ucas.ac.cn

\begin{abstract}
This paper is devoted to studying difference indices of quasi-regular difference algebraic systems. We give the definition of difference indices through a family of pseudo-Jacobian matrices. Some properties of difference indices are proved. In particular, a Jacobi-type upper bound for the sum of the difference index and the order is given. As applications of difference indices, an upper bound of the Hilbert-Levin regularity and an upper bound of the order for the difference ideal membership problem are deduced.
\end{abstract}

\section{Introduction}

The main notion we consider in this paper is difference indices. Roughly speaking, the difference index of a difference algebraic system is the order of transform we need to apply to the system to obtain the relations up to a prescribed order that all the solutions must verify. In some sense, difference indices can be regarded as a measure of the complexity of difference algebraic systems. Difference indices are also 
closely related to some other important invariants of difference algebraic systems, for example, the order and the Hilbert-Levin regularity. Moveover, difference indices can be utilized to solve the difference ideal membership problem.

The analogous notion for a differential algebraic system is the differential index, which has been extensively studied for many years. Actually, there are several definitions of differential indices of a differential algebraic system in the literature (see for instance $[4,11,13,15]$ ), which are not completely equivalent. However, the corresponding notion of difference indices for difference algebraic systems has been rarely studied yet. In [1] and [2], D'Alfonso et al. introduced the notion of $\mathfrak{P}$-differential indices for quasi-regular differential algebraic systems through a family of pseudo-Jacobian matrices. In this paper, we first give the definition of difference indices for quasi-regular difference algebraic systems, following the method used in [1] and [2]. Let us explain it in more details.

Suppose $F=\left\{f_{1}, \ldots, f_{r}\right\}$ is a set of difference polynomials, $\Delta$ is the difference ideal generated by $F$. For an element $a$ in a difference field, let $a^{[k]}=\left\{a, \sigma(a), \ldots, \sigma^{k}(a)\right\}$. Denote by $\Delta_{k}$ the algebraic ideal generated by $f_{1}^{[k-1]}, \ldots, f_{r}^{[k-1]}$. We say the system $F$ is quasi-regular if for every positive integer $k$, the Jacobian matrix of the polynomials $f_{1}^{[k-1]}, \ldots, f_{r}^{[k-1]}$ with respect to the set of variables has full row rank. Through a family of pseudo-Jacobian matrices, we can give the definition of the difference index of a quasi-regular difference algebraic system. As usual, its definition follows from a certain chain which eventually becomes stationary. In analogy with the case of $\mathfrak{P}$-differential indices in [1], the chain is established by the sequence of ranks of certain Jacobian submatrices associated with the system $F$. Assume that $F$ is quasi-regular and $\omega$ is the difference index of the system $F$. Then it turns out that for every $i \geq e-1$ ( $e$ is the highest order of $F$ ), $\omega$ satisfies: 


$$
\Delta_{i-e+1+\omega} \cap A_{i}=\Delta \cap A_{i}
$$

where $A_{i}$ is the polynomial ring in the variables with orders no more than $i$, which meets our expectation for difference indices.

This approach enables us to give an upper bound for the sum of the order and the difference index of a quasi-regular difference algebraic system. Basing on this, we can give several applications of difference indices, including an upper bound of the Hilbert-Levin regularity and an upper bound of orders for the difference ideal membership problem of a quasi-regular difference algebraic system.

The paper will be organized as follows. In Section 2, we list some basic notions from difference algebra which will be used later. In Section 3, we give the definition of quasi-regular difference algebraic systems. In Section 4, we introduce a family of pseudo-Jacobian matrices and give the definition of $\mathfrak{p}$-difference indices through studying the ranks of them. In Section 5, a Jacobi-type upper bound for the sum of the order and the $\mathfrak{p}$ difference index is given. In Section 6, several applications of $\mathfrak{p}$-difference indices are given. In Section 7, we give an example.

\section{Preliminaries}

A difference ring or $\sigma$-ring for short $(R, \sigma)$, is a commutative ring $R$ together with a ring endomorphism $\sigma: R \rightarrow R$. If $R$ is a field, then we call it a difference field, or a $\sigma$-field for short. We call $\sigma$ the transforming operator of $R$ and usually omit $\sigma$ from the notation, simply refer to $R$ as a $\sigma$-ring or a $\sigma$-field. A typical example of $\sigma$-field is the field of rational functions $\mathbb{Q}(x)$ with $\sigma(f(x))=f(x+1)$. For any $a \in R, \sigma(a)$ is called the transform of $a$. For $n \in \mathbb{N}, \sigma^{n}(a)=\sigma^{n-1}(\sigma(a))$ is called the $n$-th transform of $a$, with the usual assumption $\sigma^{0}(a)=a$. In this paper, unless otherwise specified, $K$ is always assumed to be a $\sigma$-field of characteristic 0 . 
Definition 2.1. Let $R$ be a $\sigma$-ring. An ideal $I$ of $R$ is called a $\sigma$-ideal if for $a \in R, a \in I$ implies $\sigma(a) \in I$. Suppose $I$ is a $\sigma$-ideal of $R$, then $I$ is said to be

- reflexive if $\sigma(a) \in I$ implies $a \in I$ for $a \in R$;

- $\sigma$-prime if $I$ is reflexive and a prime ideal as an algebraic ideal.

For a subset $F$ in a $\sigma$-ring, we denote by $[F]$ the $\sigma$-ideal generated by $F$. Let $K$ be a $\sigma$-field. Suppose $\mathbb{Y}=\left\{y_{1}, \ldots, y_{n}\right\}$ is a set of $\sigma$-indeterminates over $K$. Then the $\sigma$-polynomial ring over $K$ in $\mathbb{Y}$ is the polynomial ring in the variables $\mathbb{Y}, \sigma(\mathbb{Y}), \sigma^{2}(\mathbb{Y}), \ldots$ It is denoted by

$$
K\{\mathbb{Y}\}=K\left\{y_{1}, \ldots, y_{n}\right\}
$$

and has a natural $K$ - $\sigma$-algebra structure. For a $\sigma$-polynomial $f \in K\{\mathbb{Y}\}$, the order of $f$, denoted by $\operatorname{ord}(f)$, is the largest $j$ such that the variable $\sigma^{j}\left(y_{i}\right)$ appears in $f$ for some $i$.

For more details about difference algebra, please refer to [16].

\section{Quasi-Regular Difference Algebraic Systems}

Let $K$ be a $\sigma$-field. Let $a$ be an element in a $\sigma$-extension field of $K, S$ be a set of elements in a $\sigma$-extension field of $K$, and $i \in \mathbb{N}$. Denote $a^{(i)}=\sigma^{i}(a)$, $a^{[i]}=\left\{a, a^{(1)}, \ldots, a^{(i)}\right\}, S^{(i)}=\bigcup_{a \in S}\left\{a^{(i)}\right\}$ and $S^{[i]}=\bigcup_{a \in S} a^{[i]}$. For the $\sigma$-indeterminates $\mathbb{Y}=\left\{y_{1}, \ldots, y_{n}\right\}$ and $i \in \mathbb{N}$, we will treat the elements of $\mathbb{Y}^{[i]}$ as algebraic indeterminates, and $K\left[\mathbb{Y}^{[i]}\right]$ is the polynomial ring in $\mathbb{Y}^{[i]}$.

Throughout the paper, let $F=\left\{f_{1}, \ldots, f_{r}\right\} \subset K\{\mathbb{Y}\}$ be a set of difference polynomials over $K$ and $\mathfrak{p} \subseteq K\{\mathbb{Y}\}$ be a $\sigma$-prime $\sigma$-ideal 
minimal over $[F]$. Let $\epsilon_{i j}:=\operatorname{ord}_{y_{j}}\left(f_{i}\right)$ which is the order of $f_{i}$ with respect to $y_{j}$ and denote $e=\max \left\{\epsilon_{i j}\right\}$ for the maximal order of an element of $F$. We assume that $F$ actually involves the transforming operator, i.e., $e \geq 1$. For every $k \in \mathbb{N}$, let $A_{k}:=K\left[\mathbb{Y}^{[k]}\right]$ and $\Delta_{k}:=$ $\left(f_{1}^{[k-1]}, \ldots, f_{r}^{[k-1]}\right) \subseteq A_{k-1+e}$. We set $\Delta_{0}=(0)$.

For every $k \in \mathbb{N}$, we write $B_{k}$ for the local ring obtained from $A_{k}$ after localization at the prime ideal $A_{k} \cap \mathfrak{p}$ and let $\mathfrak{p}_{k}:=A_{k-1+e} \cap \mathfrak{p}$. Since each $A_{k}$ is a polynomial ring, the localizations $B_{k}$ are regular rings. For the sake of simplicity, we preserve the notation $\Delta_{k}$ for the ideal generated by $f_{1}^{[k-1]}, \ldots, f_{r}^{[k-1]}$ in the local ring $B_{k-1+e}$ and denote by $\Delta$ the $\sigma$-ideal generated by $F$ in $K\{\mathbb{Y}\}_{\mathfrak{p}}$.

Definition 3.1. We say the system $F$ is quasi-regular at $\mathfrak{p}$ if for every positive integer $k$, the Jacobian matrix of the polynomials $f_{1}^{[k-1]}, \ldots, f_{r}^{[k-1]}$ with respect to the set of variables $\mathbb{Y}^{[k-1+e]}$ has full row rank over the domain $A_{k-1+e} / \mathfrak{p}_{k}$ and $\Delta$ is reflexive.

Remark 3.2. If the $\sigma$-ideal $[F] \subseteq K\{\mathbb{Y}\}$ is already a $\sigma$-prime $\sigma$-ideal, the minimality of $\mathfrak{p}$ implies that $\mathfrak{p}=[F]$ and all our results remain true considering the rings $A_{k}$ and the $\sigma$-ideal $[F]$ without localization. In this case if $F$ is quasi-regular at $[F]$, we will say simply that $F$ is quasi-regular.

Proposition 3.3. Let $F$ be a difference algebraic system which is quasi-regular at p. For $k \in \mathbb{N}^{*}$, we have:

(1) $f_{1}^{[k-1]}, \ldots, f_{r}^{[k-1]}$ is a regular sequence in the local ring $B_{k-1+e}$ and generates a prime ideal.

(2) In the localized ring $K\{\mathbb{Y}\}_{\mathfrak{p}}$, $\Delta$ agrees with $\mathfrak{p} K\{\mathbb{Y}\}_{\mathfrak{p}}$. 
(3) If $\kappa$ denotes the residue field of $\mathfrak{p}$, the difference transcendence degree of $\kappa$ over $K$ is $n-r$.

Proof. For the proof, please refer to ([1], Proposition 3), which is for the differential case, but also suitable for the difference case.

\section{The Definition of $\mathfrak{p}$-Difference Index}

Following [1], we introduce a family of pseudo-Jacobian matrices which we need in order to define the concept of difference index. For a

matrix $E$ over $K$, we use $E^{(i)}$ to denote the matrix whose elements are the $i$-th transform of the corresponding elements of $E$.

Definition 4.1. For each $k \in \mathbb{N}$ and $i \in \mathbb{N}_{\geq e-1}$ (i.e., $i \in \mathbb{N}$ and $i \geq e-1$ ), we define the $k r \times k n$-matrix $J_{k, i}$ as follows:

$$
\begin{aligned}
J_{k, i}: & =\frac{\partial\left(F^{(i-e+1)}, F^{(i-e+2)}, \ldots, F^{(i-e+k)}\right)}{\partial\left(\mathbb{Y}^{(i+1)}, \mathbb{Y}^{(i+2)}, \ldots, \mathbb{Y}^{(i+k)}\right)} \\
& =\left(\begin{array}{cccc}
\frac{\partial F^{(i-e+1)}}{\partial \mathbb{Y}^{(i+1)}} & 0 & \cdots & 0 \\
\frac{\partial F^{(i-e+2)}}{\partial \mathbb{Y}^{(i+1)}} & \frac{\partial F^{(i-e+2)}}{\partial \mathbb{Y}^{(i+2)}} & \cdots & 0 \\
\vdots & \vdots & \ddots & \vdots \\
\frac{\partial F^{(i-e+k)}}{\partial \mathbb{Y}^{(i+1)}} & \frac{\partial F^{(i-e+k)}}{\partial \mathbb{Y}^{(i+2)}} & \cdots & \frac{\partial F^{(i-e+k)}}{\partial \mathbb{Y}^{(i+k)}}
\end{array}\right),
\end{aligned}
$$

where each $\frac{\partial F^{(p)}}{\partial \mathbb{Y}^{(q)}}$ denotes the Jacobian matrix $\left(\partial\left(f_{1}^{(p)}, \ldots, f_{r}^{(p)}\right) / \partial\right.$ $\left.\left(y_{1}^{(q)}, \ldots, y_{n}^{(q)}\right)\right)_{r \times n}$.

Since the partial derivative operator commutes with the transforming operator, we have 


$$
J_{k, i}=\left(\begin{array}{cccc}
\left(\frac{\partial F}{\partial \mathbb{Y}^{(e)}}\right)^{(i-e+1)} & 0 & \cdots & 0 \\
\left(\frac{\partial F}{\partial \mathbb{Y}^{(e-1)}}\right)^{(i-e+2)} & \left(\frac{\partial F}{\partial \mathbb{Y}^{(e)}}\right)^{(i-e+2)} & \cdots & 0 \\
\vdots & \vdots & \ddots & \vdots \\
\left(\frac{\partial F}{\partial \mathbb{Y}^{(e-k+1)}}\right)^{(i-e+k)} & \left(\frac{\partial F}{\partial \mathbb{Y}^{(e-k+2)}}\right)^{(i-e+k)} & \cdots & \left(\frac{\partial F}{\partial \mathbb{Y}^{(e)}}\right)^{(i-e+k)}
\end{array}\right),
$$

where we set that $\frac{\partial F}{\partial Y^{(j)}}=0$ if $j<0$.

Note that we have $J_{k, i+1}=J_{k, i}^{(1)}$ and

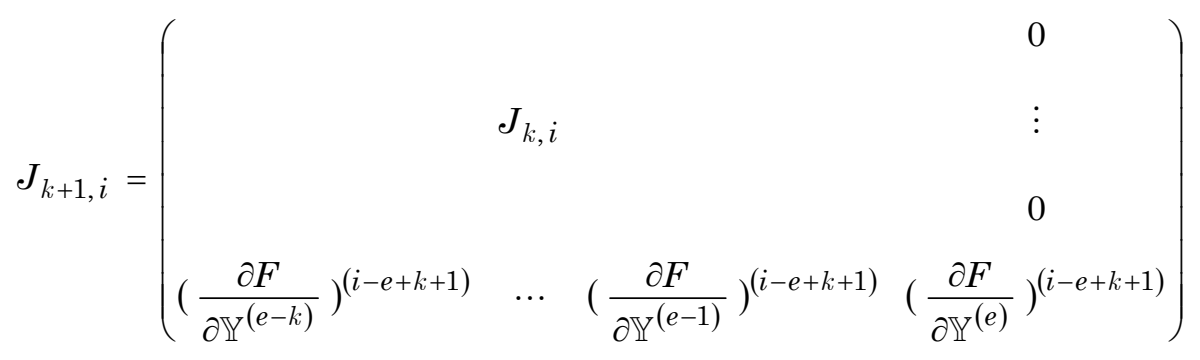

$$
=\left(\begin{array}{cccc}
\left(\frac{\partial F}{\partial \mathbb{Y}^{(e)}}\right)^{(i-e+1)} & 0 & \cdots & 0 \\
\left(\frac{\partial F}{\partial \mathbb{Y}^{(e-1)}}\right)^{(i-e+2)} & & \\
\vdots & J_{k, i}^{(1)} \\
\left(\frac{\partial F}{\partial \mathbb{Y}^{(e-k)}}\right)^{(i-e+k+1)} &
\end{array}\right)
$$

Definition 4.2. For $k \in \mathbb{N}$ and $i \in \mathbb{N}_{\geq e-1}$, we define $\mu_{k, i} \in \mathbb{N}$ as follows:

- $\mu_{0, i}:=0$

- $\mu_{k, i}:=\operatorname{dim}_{\kappa} \operatorname{ker}\left(J_{k, i}^{\tau}\right)$, for $k \geq 1$, where $J_{k, i}^{\tau}$ denotes the usual transpose of the matrix $J_{k, i}$ and $\kappa$ denotes the residue field of $\mathfrak{p}$. In particular, $\mu_{k, i}=k r-\operatorname{rank}_{\kappa}\left(J_{k, i}\right)$. 
Proposition 4.3. Let $k \in \mathbb{N}$ and $i \in \mathbb{N}_{\geq e-1}$. Then $\mu_{k, i}=\mu_{k, i+1}$.

Proof. Since $J_{k, i+1}=J_{k, i}^{(1)}$ for any $k \in \mathbb{N}$ and any $i \in \mathbb{N}_{\geq e-1}$, we just need to show that $J_{k, i}^{(1)}$ and $J_{k, i}$ have the same rank. This is clear since the maximal nonzero minors of $J_{k, i}^{(1)}$ and $J_{k, i}$ have the same order.

The previous proposition shows that the sequence $\mu_{k, i}$ does not depend on the index $i$. Therefore, in the sequel, we will write $\mu_{k}$ instead of $\mu_{k, i}$, for any $i \in \mathbb{N}_{\geq e-1}$.

For $k \in \mathbb{N}$, denote by $\kappa\left(\Delta_{k}\right)$ the residue field of $\Delta_{k}$ in the ring $B_{k-1+e}$, by $\kappa\left(\mathfrak{p}_{k}\right)$ the residue field of $\mathfrak{p}_{k}$ in the ring $A_{k-1+e}$ and by $\kappa$ the residue field of $\mathfrak{p}$. As an additional hypothesis on the system $F$, we assume that the rank of the matrix $J_{k, i}$ over $\kappa\left(\Delta_{i-e+1+k+s}\right)$ does not depend on $s$, where $s \in \mathbb{N}$. That is to say, the rank of the matrix $J_{k, i}$ considered alternatively over $\kappa\left(\Delta_{i-e+1+k}\right)$, or over $\kappa\left(\mathfrak{p}_{i-e+1+k}\right)$, or over $\kappa$ is always the same.

Proposition 4.4. Let $k \in \mathbb{N}$ and $i \in \mathbb{N}_{\geq e-1}$. Then:

(1) The transcendence degree of the field extension

$$
\operatorname{Frac}\left(B_{i} /\left(\Delta_{i-e+1+k} \cap B_{i}\right)\right) \hookrightarrow \operatorname{Frac}\left(B_{i+k} / \Delta_{i-e+1+k}\right)
$$

is $k(n-r)+\mu_{k}$.

(2) The following identity holds: $\operatorname{trdeg}_{K}\left(\operatorname{Frac}\left(B_{i} /\left(\Delta_{i-e+1+k} \cap B_{i}\right)\right)\right)=(n-r)(i+1)+e r-\mu_{k}$.

Proof. For the proof, please refer to [1, Proposition 6], which is for the differential case, but also suitable for the difference case. 
Proposition 4.5. The sequence $\left(\mu_{k}\right)_{k \in \mathbb{N}}$ is non-decreasing and verifies the inequality

$$
\sum_{j=1}^{r} \min \left\{k, e-e_{j}\right\} \leq \mu_{k} \leq \min \{k, e\} r .
$$

In particular, there exists $k \in \mathbb{N}, 0 \leq k \leq e+\sum_{j=1}^{r} e_{j}$, such that $\mu_{k}=\mu_{k+1}$.

Proof. Fix an index $i \in \mathbb{N}_{\geq e-1}$. From (1), it is easy to see that ker $\left(J_{k, i}^{\tau}\right) \times\{0\}^{r} \subseteq \operatorname{ker}\left(J_{k+1, i}^{\tau}\right)$ for every $k \in \mathbb{N}$. Then the fact $\left(\mu_{k}\right)_{k \in \mathbb{N}}$ is a non-decreasing sequence follows immediately.

For $k \in \mathbb{N}$, since the matrix $J_{k, i}$ has $k r$ rows, it is clear that $\operatorname{dim}_{\kappa}$ $\operatorname{ker}\left(J_{k, i}^{\tau}\right) \leq k r$. Due to Proposition 4.4, we have $\operatorname{trdeg}_{K}\left(\operatorname{Frac}\left(B_{i} /\right.\right.$ $\left.\left.\Delta_{i-e+1+k} \cap B_{i}\right)\right)=(n-r)(i+1)+e r-\mu_{k}$. Since $\Delta_{i-e+1+k} \cap B_{i} \subseteq \Delta \cap B_{i}$, $\operatorname{trdeg}_{K}\left(\operatorname{Frac}\left(B_{i} / \Delta_{i-e+1+k} \cap B_{i}\right)\right) \geq \operatorname{trdeg}_{K}\left(\operatorname{Frac}\left(B_{i} / \Delta \cap B_{i}\right)\right)$, and hence $\operatorname{trdeg}_{K}\left(\operatorname{Frac}\left(B_{i} / \Delta \cap B_{i}\right)\right) \leq(n-r)(i+1)+e r-\mu_{k}$. On the other hand, the fact that the difference dimension of $\Delta$ is $n-r$ implies $\operatorname{trdeg}_{K}\left(\operatorname{Frac}\left(B_{i} / \Delta \cap B_{i}\right)\right) \geq(n-r)(i+1)$. Hence, $\mu_{k} \leq e r$ holds.

In order to show the other inequality, we observe that, since the order of the polynomial $f_{j}$ is $e_{j}(1 \leq j \leq r)$, the partial derivatives $\frac{\partial f_{j}}{\partial \mathbb{Y}^{(q)}}$ are zeros for $q>e_{j}$. So, each polynomial $f_{j}$ induces $k$ null rows if $e-k$ $+1>e_{j}$ or $e-e_{j}$ null rows if $e-k+1 \leq e_{j}$ in the matrix $J_{k, i}$. Equivalently, $f_{j}$ induces $\min \left\{k, e-e_{j}\right\}$ many null rows in the matrix $J_{k, i}$. We conclude that the matrix $J_{k, i}$ has at least $\sum_{j=1}^{r} \min \left\{k, e-e_{j}\right\}$ null rows. Thus, the dimension of the kernel of the transpose matrix 
$J_{k, i}^{\tau}\left(\right.$ i.e., $\left.\mu_{k}\right)$ is at least $\sum_{j=1}^{r} \min \left\{k, e-e_{j}\right\}$. The second assertion follows directly from the fact that for every $k \geq e$, the inequality (3) reads $\sum_{j=1}^{r}\left(e-e_{j}\right) \leq \mu_{k} \leq e r$.

Theorem 4.6. Fix an index $i \in \mathbb{N}_{\geq e-1}$. Let $k_{0} \in \mathbb{N}$ be the minimum of $k$ such that $\mu_{k+1}=\mu_{k}$ (this minimum is well defined due to Proposition 4.5). Then $\mu_{k}=\mu_{k_{0}}$ for every $k \geq k_{0}$.

Proof. The case for $k_{0}=0$ is easy. In this case, $\mu_{1}=0$, which is equivalent to the fact that the matrix $J_{1, i}$ has full row rank. So $J_{k, i}$ has full row rank too. Hence $\mu_{k}=0$ for all $k$.

Now, let us assume that $k_{0} \geq 1$. It suffices to show that $\mu_{k}=\mu_{k-1}$ implies $\mu_{k+1}=\mu_{k}$ for $k \geq 2$. In the sequel, for a vector $v \in \kappa^{l r}$ we will write its description as a block vector $v=\left(v_{1}, \ldots, v_{l}\right)$ with $v_{j} \in \kappa^{r}$. Due to the recursive relation (1), the identity $\operatorname{ker}\left(J_{k, i}^{t}\right) \times\{0\}^{r}=\operatorname{ker}\left(J_{k+1, i}^{t}\right) \cap$ $\left\{v_{k+1}=\mathbf{0}\right\}$ holds in $\kappa^{(k+1) r}$ for every $k \in \mathbb{N}$ and so, the equality $\mu_{k}=\mu_{k+1}$ is equivalent to the inclusion $\operatorname{ker}\left(J_{k+1, i}^{t}\right) \subseteq\left\{v_{k+1}=\mathbf{0}\right\}$. Then, the theorem is a consequence of the following recursive principle:

Claim: For all $k \in \mathbb{N}, \operatorname{ker}\left(\boldsymbol{J}_{k, i}^{\tau}\right) \subseteq\left\{v_{k}=\mathbf{0}\right\}$ implies $\operatorname{ker}\left(\boldsymbol{J}_{k+1, i}^{\tau}\right) \subseteq$ $\left\{v_{k+1}=\mathbf{0}\right\}$.

Proof of the claim. Suppose $w=\left(w_{1}, \ldots, w_{k+1}\right)^{\tau}$ is a solution of $J_{k+1, i}^{\tau}$, then by the recursive relation (2), we have $\left(w_{2}, \ldots, w_{k+1}\right) \cdot J_{k, i}^{(1)}=0$. Since $\operatorname{ker}\left(J_{k, i}^{\tau}\right) \subseteq\left\{v_{k}=\mathbf{0}\right\}, J_{k, i}^{\tau}$ can be transformed to a reduced row echelon matrix with the last $k$ rows and the last $k$ columns forming an 
identity matrix through the elementary row transformations, and so can $\left(J_{k, i}^{(1)}\right)^{\tau}$. It follows $w_{k+1}=\mathbf{0}$ which proves the claim.

Definition 4.7. By Theorem 4.6, there exists $\omega \in \mathbb{N}$ such that $\mu_{k}<\mu_{k+1}$ for all $k<\omega$ and $\mu_{k}=\mu_{k+1}$ for all $k \geq \omega$. Such $\omega$ is called the $\mathfrak{p}$-difference index of the system $F$. If $[F]$ is itself a $\sigma$-prime $\sigma$-ideal, we say simply the difference index of $F$.

It is obvious from the construction that $\omega$ is depending on the choice of $\mathfrak{p}$. However, we will prove some properties of $\omega$ which meet our expectation for difference indices.

\section{Properties of $\mathfrak{p}$-Difference Index}

A notable property associated with most differentiation indices is that they provide an upper bound for the number of derivatives of the system needed to obtain all the equations that must be satisfied by the solutions of the system. This case is also suitable for the $\mathfrak{p}$-difference indices defined above.

Theorem 5.1. Suppose that $F$ is a difference algebraic system which is quasi-regular at $\mathfrak{p}$ and let $\omega$ be the $\mathfrak{p}$-difference index of the system $F$. Then, for every $i \in \mathbb{N}_{\geq e-1}$, the equality of ideals

$$
\Delta_{i-e+1+\omega} \cap B_{i}=\Delta \cap B_{i}
$$

holds in the ring $B_{i}$. Furthermore, for every $i \in \mathbb{N}_{\geq e-1}$, the $\mathfrak{p}$-difference index $\omega$ verifies: $\omega=\min \left\{h \in \mathbb{N}: \Delta_{i-e+1+h} \cap B_{i}=\Delta \cap B_{i}\right\}$.

Proof. Fix an index $i \in \mathbb{N}_{\geq e-1}$. Consider the increasing chain $\left(\Delta_{i-e+1+k} \cap B_{i}\right)_{k \in \mathbb{N}}$ of prime ideals in the ring $B_{i}$. For every $k \in \mathbb{N}$, by Proposition 4.4, 


$$
\operatorname{trdeg}_{K}\left(\operatorname{Frac}\left(B_{i} /\left(\Delta_{i-e+1+k} \cap B_{i}\right)\right)\right)=(n-r)(i+1)+e r-\mu_{k} .
$$

Since when $k \geq \omega, \mu_{k}$ is constant, we know that all the prime ideals $\Delta_{i-e+1+k} \cap B_{i}$ have the same dimension for $k \geq \omega$. It follows that $\left(\Delta_{i-e+1+k} \cap B_{i}\right)_{k \in \mathbb{N}}$ becomes stationary for $k \geq \omega$.

Next we show that the largest ideal of the chain coincides with $\Delta \cap B_{i}$. One inclusion is obvious. For the other, let $f \in \Delta \cap B_{i}$. There exist difference polynomials $h, a_{l j} \in K\{\mathbb{Y}\}, h \notin \mathfrak{p}$ such that

$$
f=\sum_{l=1}^{r} \sum_{j} \frac{a_{l j} f_{l}^{(j)}}{h} .
$$

Let $N$ be the maximal order of the variables $\mathbb{Y}$ appearing in this equality. Then we have $f \in \Delta_{N-e+1} \subseteq B_{N}$ and hence $f \in \Delta_{N-e+1} \cap B_{i}$. Since the above chain of ideals is stationary for $k \geq \omega, f \in \Delta_{i-e+1+\omega} \cap$ $B_{i}$.

In order to prove the second assertion of the theorem, let $h_{i}$ be the smallest non-negative integer such that $\Delta_{i-e+1+h_{i}} \cap B_{i}=\Delta \cap B_{i}$ for $i \in \mathbb{N}_{\geq e-1}$. By the definition of $h_{i}$, the transcendence degrees $\operatorname{trdeg}_{K}\left(\operatorname{Frac}\left(B_{i} /\left(\Delta_{i-e+1+k} \cap B_{i}\right)\right)\right)$ coincide for $k \geq h_{i}$, and hence by (4), $\mu_{k}$ is constant for $k \geq h_{i}$. This implies $\omega \leq h_{i}$. Clearly $h_{i} \leq \omega$ by the minimality of $h_{i}$. So $\omega=h_{i}$.

The following proposition reveals a connection between $\mu_{k}$ and the order of $\mathfrak{p}$.

Proposition 5.2. Assume that $F$ is a difference algebraic system which is quasi-regular at $\mathfrak{p}$ and $\omega$ is the $\mathfrak{p}$-difference index of $F$. Then $\operatorname{ord}(\mathfrak{p})=e r-\mu_{\omega}$.

Proof. Fix an index $i \in \mathbb{N}_{\geq e-1}$. By Theorem 5.1, for $k \geq \omega, \Delta_{i-e+1+k}$ $\cap B_{i}=\Delta \cap B_{i}$. Therefore, by Proposition 4.4 and Theorem 4.6, for $k \geq \omega$, 


$$
\begin{aligned}
\operatorname{trdeg}_{K}\left(\operatorname{Frac}\left(B_{i} /\left(\Delta \cap B_{i}\right)\right)\right) & =\operatorname{trdeg}_{K}\left(\operatorname{Frac}\left(B_{i} /\left(\Delta_{i-e+1+k} \cap B_{i}\right)\right)\right) \\
& =(n-r)(i+1)+e r-\mu_{k} .
\end{aligned}
$$

On the other hand, since $\operatorname{Frac}\left(B_{i} /\left(\Delta \cap B_{i}\right)\right)=\operatorname{Frac}\left(A_{i} /\left(\mathfrak{p} \cap A_{i}\right)\right)$, then by the property of the dimension polynomial of $\mathfrak{p}$ (see for instance [16], Chapter 5),

$$
\begin{aligned}
\operatorname{trdeg}_{K}\left(\operatorname{Frac}\left(B_{i} /\left(\Delta \cap B_{i}\right)\right)\right) & =\sigma-\operatorname{dim}(\mathfrak{p})(i+1)+\operatorname{ord}(\mathfrak{p}) \\
& =(n-r)(i+1)+\operatorname{ord}(\mathfrak{p}),
\end{aligned}
$$

where $\sigma-\operatorname{dim}(\mathfrak{p})=n-r$ is from Proposition 3.3. It follows $\operatorname{ord}(\mathfrak{p})=e r-\mu_{\omega}$.

Jacobi introduced a parameter associated with the orders of derivations in a differential algebraic system (known as Jacobi number) and conjectured that the order of the system was bounded by this number. Cohn generalized this to difference algebraic systems ([10]).

For a difference algebraic system $F$, we introduce an auxiliary integer matrix $\mathcal{E}_{0}:=\left(\epsilon_{i j}\right)_{r \times n}$ whose entries are the orders $\epsilon_{i j}$ of $f_{i}$ with respect to the variable $y_{j}$ appearing in $f_{i}$ and 0 if the variable $y_{j}$ does not appear in $f_{i}$.

Definition 5.3. Let $A \in \mathbb{N}^{r \times n}, r \leq n$, be an integer matrix. The Jacobi number of $A$ is defined to be

$$
J(A):=\max \left\{\sum_{i=1}^{r} a_{i \tau(i)} \mid \tau:\{1, \ldots, r\} \rightarrow\{1, \ldots, n\} \text { is an injection }\right\}
$$

We have the following the Jacobi-type bound for the sum of the $\mathfrak{p}$-difference index and the order of $\mathfrak{p}$. Since the proof is almost identical to ([1], Theorem 15), we will omit it. 
Theorem 5.4. Suppose $F$ is a difference algebraic system which is quasi-regular at $\mathfrak{p}$. Then, the $\mathfrak{p}$-difference index $\omega$ of the system $F$ and the $\operatorname{order} \operatorname{ord}(\mathfrak{p})$ of $\mathfrak{p}$ satisfy

$$
\omega+\operatorname{ord}(\mathfrak{p}) \leq J\left(\mathcal{E}_{0}\right)+e-\min \left\{\epsilon_{i j}\right\} .
$$

\section{Applications of $\mathfrak{p}$-Difference Index}

\subsection{The Hilbert-Levin regularity}

For a $\sigma$-prime $\sigma$-ideal $\mathfrak{p}$, the polynomial $\varphi(i)=\sigma-\operatorname{dim}(\mathfrak{p})(i+1)+\operatorname{ord}(\mathfrak{p})$ is known as the dimension polynomial of $\mathfrak{p}$ (see for instance [16], Chapter 5). The minimum of the indices $i_{0}$ such that $\varphi(i)=\operatorname{trdeg}_{K}$ $\left(\operatorname{Frac}\left(A_{i} /\left(A_{i} \cap \mathfrak{p}\right)\right)\right)$ for all $i \geq i_{0}$ is called the Hilbert-Levin regularity of $\mathfrak{p}$. The results developed on $\mathfrak{p}$-difference indices enable us to give an upper bound of the Hilbert-Levin regularity of $\mathfrak{p}$.

Theorem 6.1. Suppose $F$ is a difference algebraic system which is quasi-regular at $\mathfrak{p}$. Then the Hilbert-Levin regularity of the $\sigma$-prime $\sigma$-ideal $\mathfrak{p}$ is bounded by $e-1$.

Proof. Since for all $i \in \mathbb{N}$, we have $\operatorname{Frac}\left(A_{i} /\left(A_{i} \cap \mathfrak{p}\right)\right)=\operatorname{Frac}\left(B_{i} /\right.$ $\left.\left(B_{i} \cap \Delta\right)\right)$. Therefore, $\operatorname{trdeg}_{K}\left(\operatorname{Frac}\left(A_{i} /\left(A_{i} \cap \mathfrak{p}\right)\right)\right)=\operatorname{trdeg}_{K}\left(\operatorname{Frac}\left(B_{i} /\right.\right.$ $\left.\left.\left(B_{i} \cap \Delta\right)\right)\right)$ and so, it is enough to show that, for all $i \geq e-1$, $\operatorname{trdeg}_{K}\left(\operatorname{Frac}\left(B_{i} /\left(B_{i} \cap \Delta\right)\right)\right)+\sigma-\operatorname{dim}(\mathfrak{p})=\operatorname{trdeg}_{K}\left(\operatorname{Frac}\left(B_{i+1} /\left(B_{i+1} \cap \Delta\right)\right)\right)$.

Fix an index $i \geq e-1$. Let $\omega$ be the $\mathfrak{p}$-difference index of the system $F$. By Theorem 5.1, we have that $\Delta \cap B_{i}=\Delta_{i-e+1+\omega} \cap B_{i}$ and $\Delta \cap B_{i+1}$ $=\Delta_{i-e+2+\omega} \cap B_{i+1}$. Thus, by Proposition 4.4, we obtain:

$$
\operatorname{trdeg}_{K}\left(\operatorname{Frac}\left(B_{i+1} /\left(\Delta \cap B_{i+1}\right)\right)\right)=(n-r)(i+2)+e r-\mu_{\omega},
$$




$$
\operatorname{trdeg}_{K}\left(\operatorname{Frac}\left(B_{i} /\left(\Delta \cap B_{i}\right)\right)\right)=(n-r)(i+1)+e r-\mu_{\omega} .
$$

Hence, the result holds.

\subsection{The ideal membership problem}

It is well known that in polynomial algebra, the ideal membership problem is to decide if a given element $f \in A$ belongs to a fixed ideal $I \subseteq A$ for a polynomial ring $A$, and if the answer is yes, representing $f$ as a linear combination with polynomial coefficients of a given set of generators of $I$.

The ideal membership problem also exists in differential algebra and difference algebra. But unlike the case in polynomial algebra, this problem is undecidable for arbitrary ideals in differential algebra (see [7]) and difference algebra. However, there are special classes of differential ideals for which the problem is decidable, in particular, the class of radical differential ideals ([14], see also [3]).

When it comes to the representation problem, the differential case or the difference case involves another additional ingredient: the minimal number $N$ such that a given element $f$ of a differential or difference ideal $I$ can be written as a polynomial linear combination of the generators and their derivations or transforms up to the order $N$. The known order bounds seem to be too big, even for radical ideals (see for instance [8], where an upper bound in terms of the Ackerman function is given, or [9], a better and more explicit upper bound). In [1], an order bound for quasiregular differential algebraic systems is given, due to the properties of differential indices defined in the same paper. Now by virtue of Theorem 5.1 , we are able to give an order bound for the membership problem of a quasi-regular difference algebraic system.

The following ideal membership theorem for polynomial rings will be used. 
Theorem 6.2 ([5], Theorem 5.1). Let $K$ be a field and $g_{1}, \ldots, g_{s} \in K\left[y_{1}, \ldots, y_{n}\right]$ be a complete intersection of polynomials whose total degrees are bounded by an integer d. Let $g \in K\left[y_{1}, \ldots, y_{n}\right]$ be another polynomial. Then the following conditions are equivalent:

(1) $g$ belongs to the ideal generated by $g_{1}, \ldots, g_{s}$;

(2) there exist polynomials $a_{1}, \ldots, a_{s}$ such that $g=\sum_{j=1}^{s} a_{j} g_{j}$ and $\operatorname{deg}\left(a_{j} g_{j}\right) \leq d^{s}+\operatorname{deg}(g)$ for $1 \leq j \leq s$.

We have the following effective ideal membership theorem for quasiregular difference algebraic systems.

Theorem 6.3. Suppose $F$ is a quasi-regular difference algebraic system in the sense of Remark 3.2. Let D be an upper bound for the total degrees of $f_{1}, \ldots, f_{r}$. Let $f \in K\{\mathbb{Y}\}$ be an arbitrary difference polynomial in the difference ideal $[F]$. Set $N=\omega+\max \{-1, \operatorname{ord}(f)-e\}$, where $\omega$ is the difference index of $F$. Then, a representation

$$
f=\sum_{1 \leq i \leq r, 0 \leq j \leq N} g_{i j} f_{i}^{(j)}
$$

holds in the ring $A_{N+e}$, where each polynomial $g_{i j} f_{i}^{(j)}$ has total degree bounded by $\operatorname{deg}(f)+D^{r(N+1)}$.

Proof. The upper bound on the order of transforms of $f_{1}, \ldots, f_{r}$ is immediate from Theorem 5.1 by setting $i=\max \{e-1, \operatorname{ord}(f)\}$. The degree upper bound for the polynomials $g_{i j} f_{i}^{(j)}$ follows from Proposition 3.3 and Theorem 6.2.

Remark 6.4. From Theorems 5.1 and 5.4, we have for every $i \in \mathbb{N}_{\geq e-1}$, the equality $\Delta_{i+1+J\left(\mathcal{E}_{0}\right)-\min \left\{\epsilon_{i j}\right\}} \cap A_{i}=\Delta \cap A_{i}$ holds. So it 
suffices to take $N=J\left(\mathcal{E}_{0}\right)-\min \left\{\epsilon_{i j}\right\}+\max \{\operatorname{ord}(f), e-1\}$ to get more explicit upper bounds of the order and the degree in the above ideal membership problem.

\section{An Example}

Example 7.1. Notations follow as before. Consider the difference algebraic system $F=\left\{y_{1}^{(1)}-y_{1} y_{3}, y_{2}^{(1)}-y_{2} y_{3}, y_{1}+y_{2}-1\right\} \subseteq A=K\left\{y_{1}\right.$, $\left.y_{2}, y_{3}\right\}$. Then $\Delta=[F]$ is a $\sigma$-prime $\sigma$-ideal and $F$ is quasi-regular in the sense of Remark 3.2. The corresponding matrices $J_{k, 0}, k=1,2,3, \ldots$ are

$$
\begin{aligned}
& \left(\begin{array}{cccccc}
1 & 0 & 0 & & & \\
0 & 1 & 0 & & & \\
0 & 0 & 0 & & & \\
-1 & 0 & -y_{1} & 1 & 0 & 0 \\
0 & -1 & -y_{2} & 0 & 1 & 0 \\
1 & 1 & 0 & 0 & 0 & 0
\end{array}\right. \\
& \begin{array}{llllll}
-1 & 0 & -y_{1} & 1 & 0 & 0
\end{array} \\
& \begin{array}{llllll}
0 & -1 & -y_{2} & 0 & 1 & 0
\end{array} \\
& \begin{array}{llllll}
1 & 1 & 0 & 0 & 0 & 0
\end{array} \\
& \begin{array}{llllll}
-1 & 0 & -y_{1} & 1 & 0 & 0
\end{array} \\
& \begin{array}{lllllll}
0 & -1 & -y_{2} & 0 & 1 & 0
\end{array} \\
& \begin{array}{llllll}
1 & 1 & 0 & 0 & 0 & 0
\end{array}
\end{aligned}
$$


Since $y_{1}^{(i)}=y_{1}, y_{2}^{(i)}=y_{2}, y_{3}^{(i)}=1$ in the ring $A / \Delta$ for all $i \in \mathbb{N}$, we have replaced $y_{1}^{(i)}, y_{2}^{(i)}, y_{3}^{(i)}$ by $y_{1}, y_{2}, 1$, respectively in $J_{k, 0}$ for all $i \in \mathbb{N}$. It can be computed that $\operatorname{rank}\left(J_{1,0}\right)=2, \operatorname{rank}\left(J_{2,0}\right)=4$, $\operatorname{rank}\left(J_{3,0}\right)=7$, so $\mu_{1}=1, \mu_{2}=2, \mu_{3}=2$ and hence the difference index of the system $F$ is $\omega=2$. One can check that $\Delta_{2} \cap A_{0}=\Delta \cap A_{0}$.

The matrix $\mathcal{E}_{0}=\left(\begin{array}{lll}1 & 0 & 0 \\ 0 & 1 & 0 \\ 0 & 0 & 0\end{array}\right)$. Therefore, by Theorem 5.4, $\omega+\operatorname{ord}(\Delta)$

$\leq J\left(\mathcal{E}_{0}\right)+e-\min \left\{\epsilon_{i j}\right\}=2+1-0=3$. On the other hand, by Proposition 5.2, we have $\operatorname{ord}(\Delta)=e r-\mu_{\omega}=3-2=1$. Therefore, $\omega+\operatorname{ord}(\Delta)=3$, which coincides with the above upper bound.

\section{References}

[1] L. D'Alfonso, G. Jeronimo, G. Massaccesi and P. Solernó, On the index and the order of quasi-regular implicit systems of differential equations, Linear Algebra Appl. 430(8-9) (2009), 2102-2122.

[2] L. D'Alfonso, G. Jeronimo and P. Solernó, A linear algebra approach to the differential index of generic DAE systems, Appl. Algebra Engrg. Comm. Comput. 19(6) (2008), 441-473.

[3] F. Boulier, D. Lazard, F. Ollivier and M. Petitot, Representation for the radical of a finitely generated differential ideal, Proc. of ISSAC (1995), 158-166.

[4] S. Campbell and W. Gear, The index of general nonlinear DAEs, Numer. Math. 72 (1995), 173-196.

[5] A. Dickenstein, N. Fitchas, M. Giusti and C. Sessa, The membership problem for unmixed ideals is solvable in single exponential time, Discr. Appl. Math. 33 (1991), 73-94.

[6] D. Eisenbud, Commutative Algebra with a View Toward Algebraic Geometry, Springer-Verlag, New York, 2004.

[7] G. Gallo, B. Mishra and F. Ollivier, Some Constructions in Rings of Differential Polynomials, Proc. of AAECC-9, Lecture Notes in Computer Science, Springer-Verlag 539 (1991), 171-182. 
[8] O. Golubitski, M. Kondratieva, A. Ovchinnikov and A. Szanto, A bound for orders in differential Nullstellensatz, J. Algebra 322(11) (2009), 3852-3877.

[9] R. Gustavson, M. Kondratieva and A. Ovchinnikov, New effective differential Nullstellensatz, Adv. Math. 290 (2016), 1138-1159.

[10] B. A. Lando, Jacobi's bound for first order difference equations, Proc. Amer. Math. Soc. 52 (1972), 8-12.

[11] G. Le Vey, Differential Algebraic Equations: A new look at the index, Rapp. Rech. 2239, INRIA, 1994.

[12] A. Levin, Difference Algebra, Springer-Verlag, New York, 2008.

[13] C. Pantelides, The consistent inicialization of differential-algebraic equations, Sci. and Stat. Comput. 9(2) (1988), 213-231.

[14] A. Seidenberg, An elimination theory for differential algebra, Univ. California Publ. Math. (1956), 31-38.

[15] W. Seiler, Indices and solvability of general systems of differential equations, Comput. Algebra in Scientific Comput., CASC 99, Springer (1999), 365-385.

[16] M. Wibmer, Algebraic Difference Equations, Preprint, 2013. 